\title{
A044 Airborne geophysical surveys applied to urban/brownfield investigations
}

D. BEAMISH, R.J. CUSS AND E. TARTARAS

British Geological Survey, Keyworth, Nottingham, NG12 5GG, UK

\begin{abstract}
A number of airborne geophysical surveys have now been conducted in the UK using a fixedwing system operated jointly by the British and Finnish Geological Surveys. The system provides magnetic, radiometric and frequency domain electromagnetic survey measurements. Regulatory survey permissions force a variety of flight elevations typically between 54 to 244 $\mathrm{m}$. On the fringes of major conurbations, we encounter urban greenspace and brownfield areas. These areas may contain mixtures of infrastructure and built structures together with derelict and green areas. In order to provide valid interpretations of the airborne data, detailed studies of the behaviour of the data to a variety of influences found in the urban fringe of a town have been made. Non-geophysical spatial data sets are increasingly being applied to aid both the processing and interpretation of the survey data. This is particularly true in non-rural areas. Examples from a high-resolution survey acquired across a $5 \times 5 \mathrm{~km}$ coastal area to the west of Irvine, Scotland are considered.
\end{abstract}

\section{Airborne Surveying in the UK}

New high-resolution airborne geophysical coverage of the UK is to be provided by a fixedwing surveying system developed from the existing facility described by Poikonen et al. (1998). The intention is to perform surveys at a flight line spacing of $200 \mathrm{~m}$ and at low altitude (typically no greater than $90 \mathrm{~m}$ ). Our ability to perform surveys is subject to regulatory conditions in an often-crowded airspace. In relation to survey height, regulatory requirements are tied to the density of structures/dwellings below each flight line and these force a variety of flight elevations typically between minima of 54 to $244 \mathrm{~m}$ (defined as 180' and 800 '). The lower value is usually applied in the absence of structures, while the upper value must be achieved when over flying built-up areas. On the fringes of major conurbations, we encounter urban greenspace and brownfield areas. These areas may contain mixtures of infrastructure and built structures together with derelict and green areas. Our ability to obtain valid geophysical data from, at least portions of, such areas is a topic of current research. In order to assist processing/interpretation procedures, the manner in which existing nongeophysical data sets (mapping databases and GIS datasets) can be used, is being investigated.

\section{The Ardeer Survey}

As an adjunct to a larger scale survey in SW Scotland, the 5 x $5 \mathrm{~km}$ Ardeer survey was flown using $50 \mathrm{~m} \mathrm{~N}$-S flight lines. The surface relief of the survey area is shown in Figure 1. Elevations range from 0 (sea-level) to $40 \mathrm{~m}$. The letter $\mathrm{R}$ indicates a railway traversing the area partly as an embankment. The source of the surface relief data is described later. Four rectangular areas, each of $500 \times 500 \mathrm{~m}$ are shown. Maps at a scale 1:10,000 for these four zones are shown in Figure 2 to indicate the type of structures and infrastructures being 
assessed as part of the survey. The local council has a redevelopment plan in place for the entire survey area shown in Figure 1.

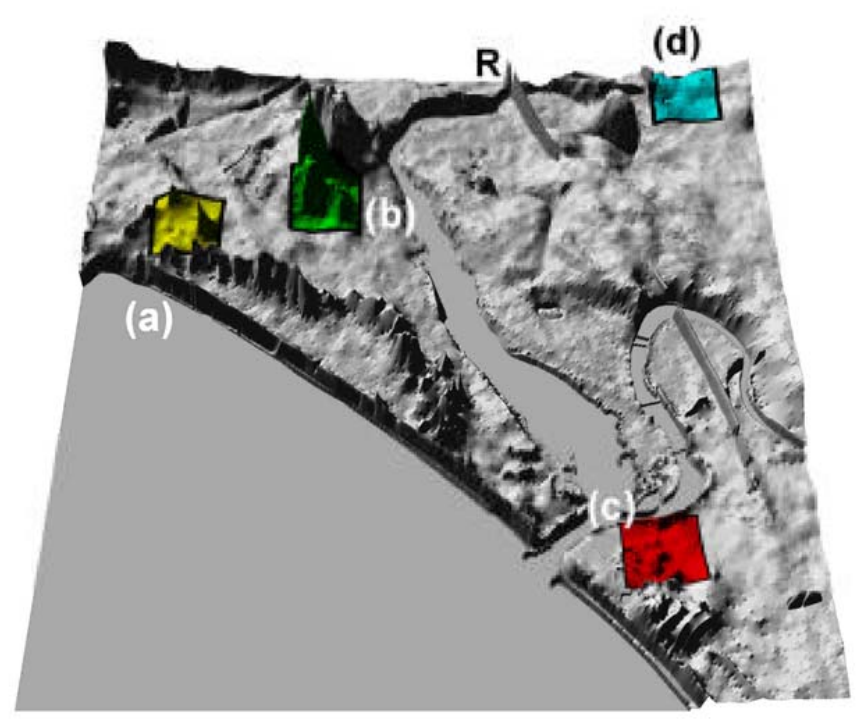

Figure 1. Surface relief of Ardeer survey area shown as 3D perspective plot. 5 x $5 \mathrm{~km}$. Range of elevation is from 0 (sea-level) to $40 \mathrm{~m}$. R denotes railway, partly on embankment. Four coloured zones (a to d) are selected 500 x $500 \mathrm{~m}$ squares areas.
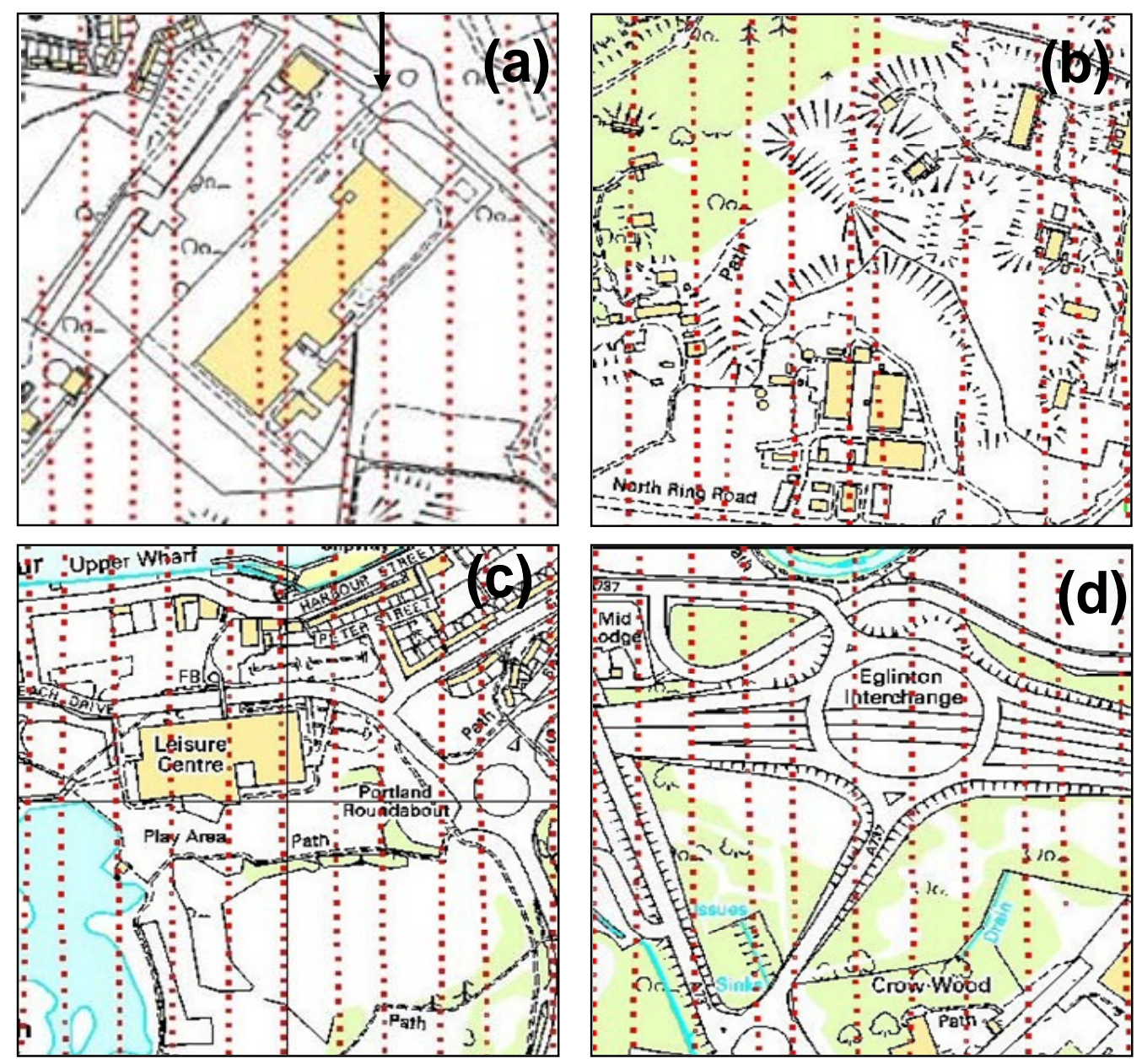

Figure 2. Four 500x500 m selected areas showing 1:10,000 maps (CCrown copyright. All rights reserved) of survey landforms. Red dots denote survey lines sampled at $4 \mathrm{~Hz}$. Arrow in (a) indicates Line 218. 
The four areas shown in Figure 2 serve as selected examples of the challenges facing the processing and interpretation of all three geophysical data sets in urban and brownfield/greenspace areas. The flight lines and $4 \mathrm{~Hz}$ sampling of the electromagnetic data are indicated by the red dots. The lateral scale of elevated structures encountered ranges from the very large (factory in Fig. 2a) to that of a modern leisure centre (Fig. 2c) to typical smaller scale industrial units (Fig. 2b). Major roads and associated service routes typically form perimeters to many sites (Fig. 2d). In essence such airborne surveys provide unrestricted access (within the regulatory height limitations) to a range of regeneration sites that would benefit from geophysical assessments. The challenge is essentially to identify/separate the cultural artefacts within these types of datasets.

\section{Non-geophysical data}

Two types of non-geophysical data have already been presented. The surface relief map is obtained using accurate DTM (Digital Terrain Model) and DSM (Digital Surface Model) data available for the majority of the UK. The data has a lateral cell resolution of $5 \times 5 \mathrm{~m}$ and the quoted vertical accuracies are of the order of $1 \mathrm{~m}$. This scale of information is well suited to the sampling scale of the airborne survey data. The surface relief model shown in Figure 1 is obtained by subtracting the DTM from the DSM data. The subtraction defines the heights of all cultural and vegetation features across the land surface.

Other digital products include the familiar geo-referenced raster maps such as those at 1:10,000 shown in Figure 2. Products, more suited to airborne database spatial processing, include vector (dxf) format products at scales ranging from 1:1250 (urban) to 1:10,000 (remote areas). These detailed maps identify both man-made and natural features, and are continuously updated. A further digital product is ADDRESS-POINT (C), a dataset that uniquely defines and locates all residential, business and public postal addresses in Great Britain. The use of these data to identify the locations of probable cultural/non-cultural influences on airborne geophysical data is under consideration.

\section{An example}

One requirement in airborne data processing is to determine the height of each sensor above the ground surface. The issue is more acute when low-level surveying is considered. Conventionally, this is achieved using a radar or laser altimeter. Both of these are unreliable (biased to low values) when built structures are encountered. They can also be unreliable over tree canopy (Beamish, 2002). The 3D location of the aircraft (and sensors) is achieved using modern differential GPS acquisition and processing. The vertical location (GPS-Z) is referenced to a global geoid (e.g. WGS94) that can be converted to the same local geoid used by a DTM. By merging geophysical, DTM and DSM databases, it is possible to obtain an estimate sensor height (above ground) unbiased by relief. Data from the flight-line highlighted (arrow) in Fig. 2a that traverses the large factory is used as an example. Figure 3a shows the radar altitude (line with symbols), the surface relief (0-10 m, shaded) and the revised survey height calculated as above. The surface relief defines the main central factory and a second building. The influence of the main structure indicates an error of about $10 \mathrm{~m}$ on the radar altitude. The influence of the structure on half-space apparent conductivities at two frequencies (calculated after Beamish, 2002) is shown in Fig. 3b.

The structure also provides a significant magnetic anomaly. This is most readily identified in the cross-line horizontal magnetic gradient obtained using the two wingtip magnetometers. 
The horizontal gradient observed is shown in Fig. 3c. The perturbation extends over a lateral distance of $\sim 500 \mathrm{~m}$.
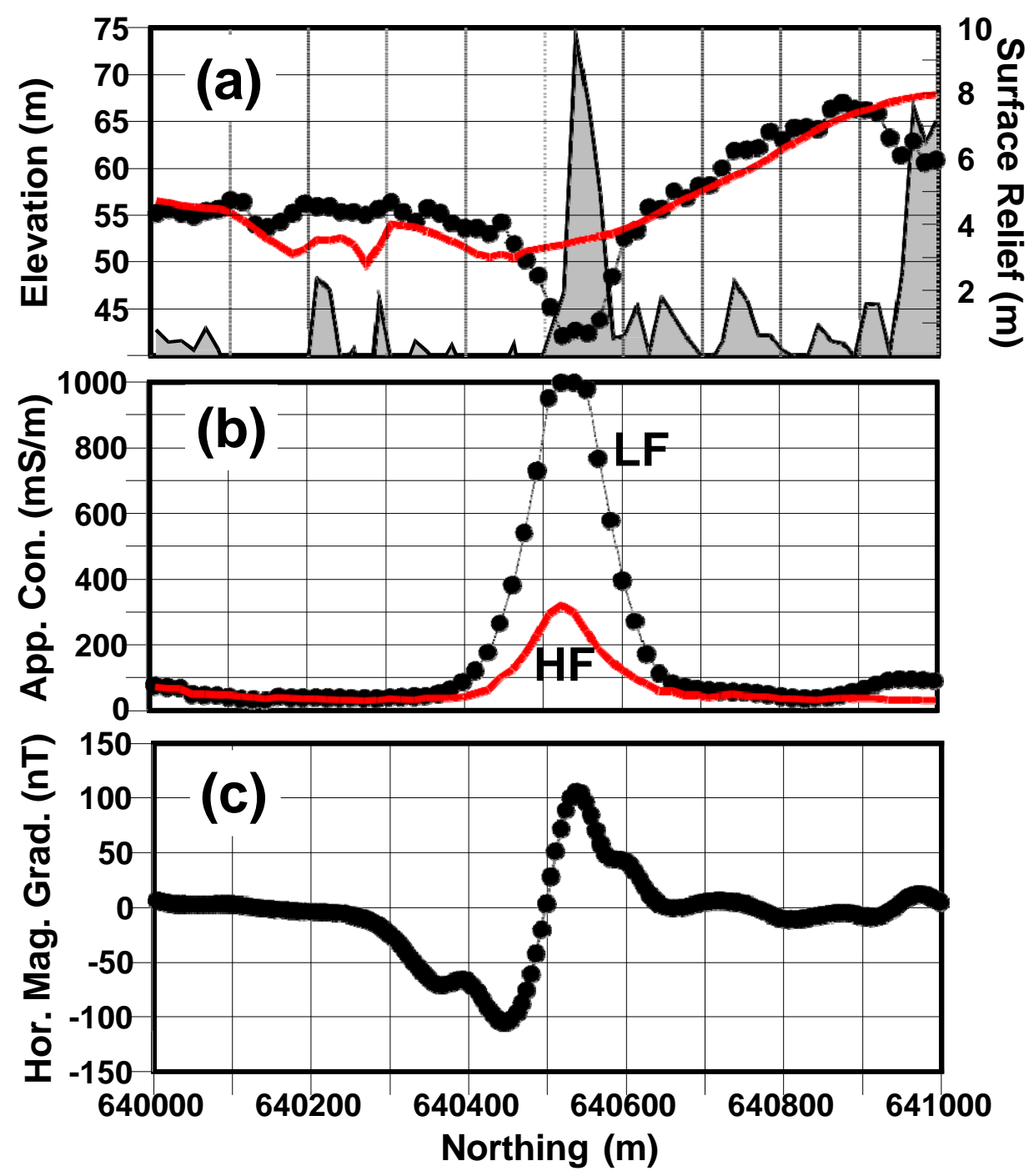

Figure 3. Line 218 data over 1 km, centred on building in Fig.2a. (a) Elevation data from radar altimeter (line with symbols), GPS/DTM corrected elevation (red line), surface relief (infill) from DTM/DSM. (b)Half-space apparent conductivities at $3 \mathrm{kHz}$ (LF) and $14 \mathrm{kHz}$ (HF). (c) measured horizontal magnetic gradient across flight line.

\section{Conclusions}

Airborne geophysical survey data acquired in the UK will encounter a wide range of infrastructure and cultural influences. These will be particularly acute on the fringes of conurbations where many redevelopment and environmental issues exist. The challenge is essentially to identify/separate the cultural artefacts within these types of data. Deculturing procedures already exist in the case of airborne magnetic data; we now require to enhance such procedures and extend them to the other geophysical measurements. In our opinion, nongeophysical information can significantly enhance the processing/interpretation of such data.

\section{References}

Beamish, D., 2002. The canopy effect in airborne EM. Geophysics, 67, 1720-1728.

Poikonen, A., Sulkanen, K, Oksama, M., Suppala, I., 1998. Novel dual frequency fixed wing airborne EM system of Geological Survey of Finland (GTK). Exploration Geophysics 29, 4651. 